\title{
Plantas medicinais e ritualísticas vendidas em feiras livres no Município do Rio de Janeiro, RJ, Brasil: estudo de caso nas zonas Norte e Sul ${ }^{1}$
}

\author{
Veronica Maioli-Azevedo ${ }^{2,3}$ e Viviane Stern da Fonseca-Kruel ${ }^{2}$
}

Recebido em 22/07/2005. Aceito em 18/09/2006

\begin{abstract}
RESUMO - (Plantas medicinais e ritualísticas vendidas em feiras livres no Município do Rio de Janeiro, RJ, Brasil: estudo de caso nas zonas Norte e Sul). O Município do Rio de Janeiro (RJ) possui 210 feiras livres cadastradas pela Prefeitura, destas 33 localizam-se nas zonas Norte e Sul do RJ. O presente estudo teve como objetivos conhecer as plantas medicinais e/ou ritualísticas vendidas em feiras livres no RJ, verificando suas indicações terapêuticas, posologia e procedência, valorizando o conhecimento empírico agregado dos erveiros e suas histórias de vida. Realizou-se 60 entrevistas semi-estruturadas e técnicas de observação direta e participante a 54 erveiros em 33 feiras livres. Relacionou-se 106 espécies vegetais distribuídas em 92 gêneros e 49 famílias; sendo 61 destas associadas exclusivamente ao uso medicinal, 19 ao uso ritualístico e 19 ao uso medicinal-ritual. Foram identificados múltiplos usos em duas espécies utilizadas como ritual-alimentar e quatro espécies medicinal-alimentar. As famílias mais representativas foram Asteraceae (18 espécies) e Lamiaceae (11 espécies). Em relação à procedência, $14 \%$ dos erveiros informaram que as espécies vendidas são cultivadas, $20 \%$ extraem da mata e $66 \%$ adquirem as plantas em grandes mercados do Município. Estas informações poderão subsidiar atividades que possam contribuir para melhor orientação sobre as plantas medicinais e/ou ritualísticas mais vendidas no RJ.
\end{abstract}

Palavras-chave: etnobotânica, feira livre, plantas medicinais, plantas ritualísticas

\begin{abstract}
Medicinal and ritual plants sold in street markets of Rio de Janeiro, RJ, Brazil: a case study in the North and South zones). Rio de Janeiro Municipality has 210 licensed street fairs of which 33 are located in the northern and southern zones. This study aims to identify the medicinal and ritual plants sold at the street fairs in Rio de Janeiro, verify therapeutic use, dosage and origin, and show the importance of the herb sellers's empirical knowledge and life histories. In 60 private interviews with 54 herb sellers, 106 species (92 genera; 49 families) were recorded: 61 for medicinal purposes, 19 for religious application and 19 for medicinal-religious purposes. Some species had multiple uses: two for religious-dietary purposes and four for medicinal-dietary use. The most representative families are Asteraceae (18 species) and Lamiaceae (11 species). As regards origin, $14 \%$ of the herb sellers reported that the species sold were home-grown, $20 \%$ extracted them from the forest and $66 \%$ purchased them from the large municipal open markets. This data will improve our knowledge of the medicinal and ritualistic species sold in street markets of Rio de Janeiro.
\end{abstract}

Key words: ethnobotany, street markets, medicinal plants, ritual plants

\section{Introdução}

A etnobotânica pode ser definida como o estudo das sociedades humanas, passadas e presentes, e todos os tipos de inter-relações: ecológicas, evolucionárias e simbólicas; reconhecendo a dinâmica natural das relações entre o ser humano e as plantas (Alexiades 1996). Os estudos etnobotânicos são importantes especialmente no Brasil, uma vez que seu território abriga uma das floras mais ricas do mundo, da qual 99\% são desconhecidas quimicamente (Gottlieb et al. 1998).

A utilização de plantas medicinais e rituais no Brasil é uma prática comum resultante da forte influência cultural dos indígenas locais miscigenadas as tradições africanas, oriundas de três séculos de tráfico escravo e da cultura européia trazida pelos colonizadores (Almeida 2003).

As feiras livres são um manancial, praticamente inexplorado, de investigações etnobotânicas que podem fornecer informações da maior importância para o conhecimento da diversidade, manejo e universo cultural de populações marginalizadas.

O uso e o comércio de plantas vêm sendo estimulados, nas últimas décadas, pela necessidade de uma crescente população que busca uma maior diversidade e quantidade de plantas para serem utilizadas no cuidado da saúde e também aplicadas em

\footnotetext{
1 Monografia de iniciação científica da primeira Autora

2 Instituto de Pesquisas Jardim Botânico do Rio de Janeiro, Rua Pacheco Leão 915, Jardim Botânico, 22460-030 Rio de Janeiro, RJ, Brasil

3 Autor para correspondência: maioli@jbrj.gov.br; vmaioli_az@hotmail.com
} 
tradições religiosas. No Brasil há crescente interesse e busca pela medicina tradicional e pela Fitoterapia (Almeida 2003) que ocorre devido a vigente carência de recursos dos órgãos públicos de saúde e incessantes aumentos de preços nos medicamentos alopáticos, bem como dos efeitos colaterais apresentados por alguns destes medicamentos (Parente \& Rosa 2001). É notório o interesse por tais plantas, tanto em âmbito nacional quanto internacional, pois estas apresentam um potencial terapêutico e econômico, visado especialmente pela indústria farmacêutica que realiza a prospecção de novos produtos (Camarra 1995; Simões et al. 1998; TRAFFIC 2001).

A prática da medicina tradicional está incluída e reconhecida no sistema primário de saúde em países em desenvolvimento (Akerele 1988; Bodeker 1994; Cunningham 1993; Sheldon, Balick \& Laird 1997; Balick et al. 2000). A Organização Mundial de Saúde (WHO 2002) estima que cerca de $80 \%$ da população mundial depende de plantas para o cuidado com a saúde, relatam ainda, que $85 \%$ da medicina tradicional envolvem o uso de plantas medicinais, seus extratos vegetais e seus princípios ativos (IUCN 1993). Estimase que o mercado atual de medicina tradicional movimenta 60 milhões de dólares nos EUA (OMS 2002).

Segundo Martin (1995) estudos detalhados sobre os recursos biológicos vendidos em mercados locais são fundamentais para uma pesquisa econômica completa, pois muitas plantas úteis apresentam valor estritamente regional que só pode ser descoberto a partir de conversas diretas com os produtores, consumidores e vendedores. Tais estudos são fundamentais e urgentes no Brasil, principalmente para obter informações sobre o comércio de plantas medicinais, pois o extrativismo destas é predatório e tem levado a reduções drásticas destas populações naturais, devido ao desconhecimento dos mecanismos de perpetuação das plantas medicinais nas florestas (Reis 1996).

No Brasil, as feiras livres e os mercados surgiram em 1841, como uma solução para o abastecimento regional de produtos, substituindo as bancas de pescado (Gorberg \& Fridman 2003). Nestas feiras e mercados instituídos pelo governo, só se vendiam certos artigos, em lugares específicos e com taxas estabelecidas pelo poder municipal. Os primeiros decretos já manifestavam preocupações com: a higiene dos feirantes; o respeito ao público; informações sobre os preços dos produtos e ainda com a formação de uma ética profissional (SAREM/SEPLAN-RR 1982).
Atualmente, dentre os mais importantes mercados do Rio de Janeiro estão, o Centro de Abastecimento do Estado da Guanabara (CADEG), Centro Estadual de Abastecimento (CEASA) e o Mercado de Madureira, os quais são os principais locais de abastecimento e repasse de produtos vegetais para a cidade do RJ.

Poucos são os estudos relacionados à comercialização de plantas em feiras e/ou mercados no Brasil (Berg 1984; M.M. Stalcup, dados não publicados; Santos \& Silvestre 2000; TRAFFIC 2001; Parente \& Rosa 2001; Almeida \& Albuquerque 2002; Shanley et al. 2002; Pinto \& Maduro 2003; Azevedo \& Silva 2006).

O presente estudo teve como objetivos, identificar as plantas medicinais e ritualísticas vendidas em feiras livres do Município do Rio de Janeiro; verificar as suas respectivas indicações terapêuticas, bem como analisar a importância relativa destas espécies vendidas, valorizando o conhecimento empírico agregado aos erveiros. Este trabalho é parte do projeto Flora de Importância Econômica do Rio de Janeiro que vem sendo desenvolvido em parceria com a Universidade Federal Rural do Rio de Janeiro e Instituto de Pesquisa Jardim Botânico do Rio de Janeiro.

\section{Material e métodos}

O estudo foi realizado nas feiras livres do Município do Rio de Janeiro (RJ) localizado entre as coordenadas

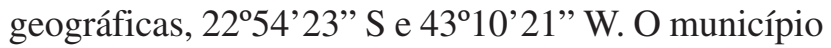
possui 1.255,3 km²; 32 Regiões Administrativas (RA) que englobam 159 bairros distribuídos nas zonas Norte, Sul, Leste e Oeste; apresenta uma população com cerca de 5.857.904 pessoas (Prefeitura do Rio de Janeiro 2003).

Para esta pesquisa selecionou-se cinco Regiões Administrativas que englobam os bairros da Tijuca, Maracanã, Vila Isabel, Andaraí, Grajaú, Rio Comprido e Estácio (zona Norte); Glória, Catete, Flamengo, Botafogo, Humaitá, Laranjeiras, Cosme Velho, Copacabana, Ipanema, Leblon, Gávea e Jardim Botânico (zona Sul) (Prefeitura do Rio de Janeiro 2003) (Fig. 1).

No período de maio/2003 a junho/2004, foram visitadas 33 feiras livres cadastradas pela Prefeitura do RJ, sendo 16 destas referentes à zona Norte, e 17 a zona Sul do Município do RJ. Nestas visitas procurouse entrevistar todos os erveiros das feiras em estudo. Logo, foram realizadas 60 entrevistas a 54 erveiros, sendo 25 do gênero masculino e 29 do gênero feminino, com idades entre 25-65 anos. Do total dos erveiros 


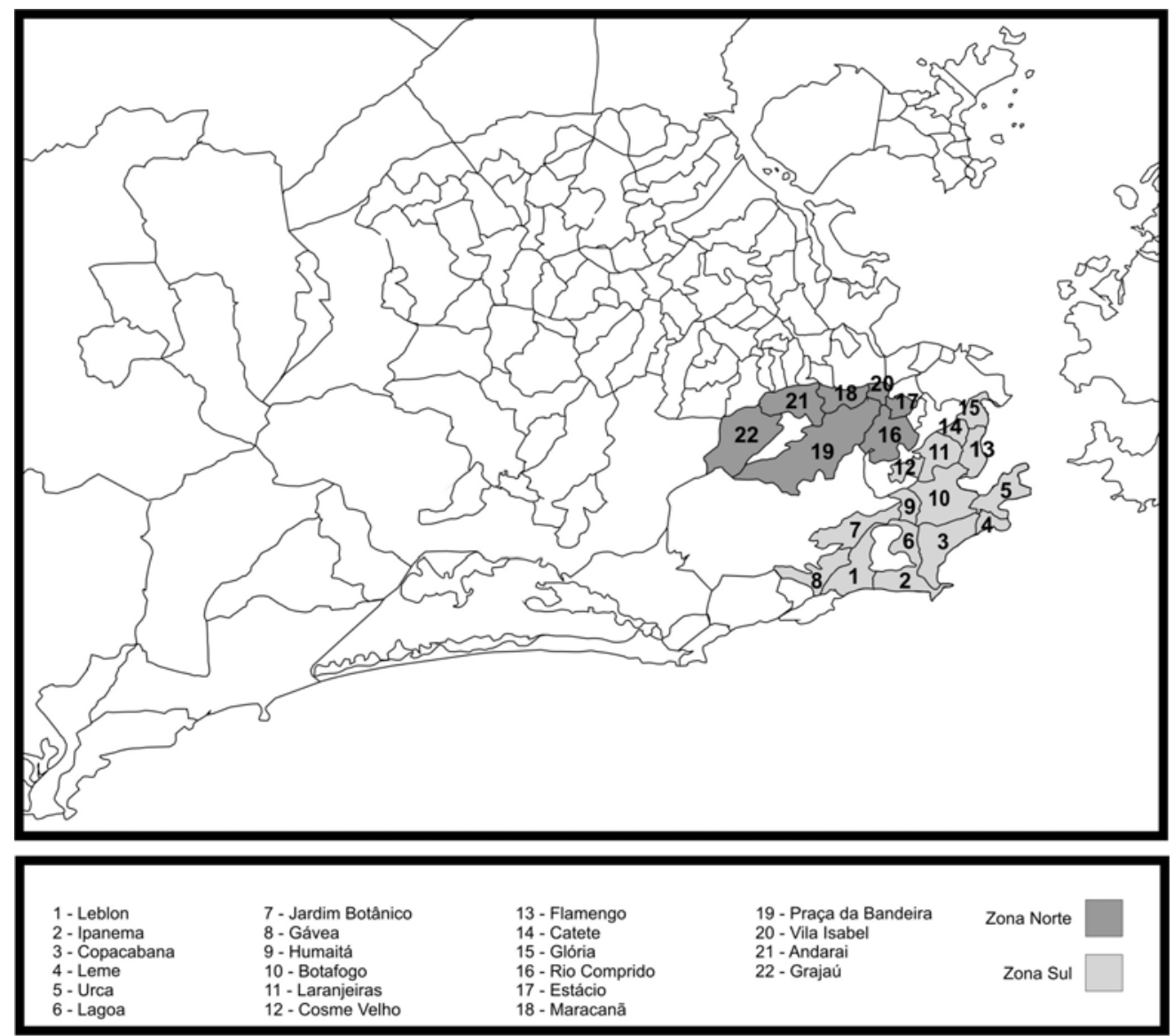

Fonte: Instituto Pereira Passos - IPP / DIG - Geoprocessamento - 1999

Figura 1. Mapa de parte do Município do Rio de Janeiro. Em destaque as Zonas Norte e Sul.

entrevistados, 24 encontram-se na zona Norte, e 30 destes na zona Sul. Técnicas de observação direta (Cotton 1996); Listagem Livre ("free listing"), adaptada de Weller \& Kimball Romney (1988) e Bernard (1989); além de questionários estruturados, com perguntas diretas e fechadas adaptadas de Alexiades (1996), foram utilizadas junto aos erveiros.

A técnica de listagem livre foi utilizada para cada informante que citou as dez plantas mais vendidas possibilitando a obtenção de informações detalhadas sobre as espécies, como principais indicações terapêuticas e partes dos vegetais mais consumidas. Os dados relacionados a partir deste levantamento foram confrontados com as espécies úteis encontradas na Lista Oficial de Espécies da Flora Brasileira Ameaçada de Extinção do IBAMA (1992), para verificar o "status" de conservação das espécies indicadas como medicinais e ritualísticas neste estudo. Ainda em relação às espécies, foram relacionadas e destacadas as nativas, das exóticas (espécies espontâneas, subespontâneas e cultivadas).

As indicações terapêuticas foram adaptadas da Classificação Estatística Internacional para Doenças e Problemas Relacionados à Saúde da Organização Mundial de Saúde (OMS 1996). Essa adaptação ocorreu devido à falta de detalhes sobre as doenças referentes às espécies indicadas, dificultando a inserção em um ou outro sistema de classificação. As espécies das quais foram obtidas maiores informações sobre o uso terapêutico foram inseridas no sistema de classificação da OMS, nas demais foram mantidos os usos informados pelos erveiros. Acrescentou-se a indicação popular relativa às plantas utilizadas em banhos ritualísticos, pois esta é uma importante categoria citada pelos erveiros, constituindo uma prática comum associada a enfermidades do espírito.

Para cada espécie de planta citada pelos informantes, calculou-se a importância relativa, com base na proposta de Bennett \& Prance (2000). O cálculo da importância relativa, sendo dois o valor 
máximo obtido por uma espécie, foi feito de acordo com a fórmula: $\mathrm{IR}=\mathrm{NSC}+\mathrm{NP}$, onde NSC $=$ número categorias terapêuticas tratadas por uma determinada espécie (NSCE), dividido pelo número total de categorias terapêuticas tratadas pela espécie mais versátil (NSCEV); NP = número de propriedades atribuídas a uma determinada espécie (NPE), dividido pelo número total de propriedades atribuídas à espécie mais versátil (NPEV).

A partir da técnica utilizada por Trotter \& Logan (1986), buscou-se identificar as indicações terapêuticas que apresentaram maior importância nas entrevistas, a partir do "consenso dos informantes". Nesta técnica os grupos de plantas merecedores de estudos são evidenciados, e para tal, utiliza-se a fórmula: $\mathrm{FCI}=$ nar-na $/$ nar -1 (onde $\mathrm{FCI}=$ fator de consenso dos informantes; nar = soma dos usos registrados por cada informante para uma categoria; e na = número de espécies indicadas para cada categoria).

Concomitantemente, a realização de cada entrevista, foi adquirido material botânico testemunho referente a cada planta citada nas entrevistas. Este foi herborizado e identificado, com base em literatura especializada, consulta a especialistas e por comparação na coleção do Herbário do JBRJ (RB), e por fim depositado nos Herbários do Jardim Botânico do Rio de Janeiro (RB) e Universidade Santa Úrsula (RUSU).

Elaborou-se um registro para cada espécie vegetal indicada pelos erveiros, contendo: nome científico, família, nome vulgar, hábito, uso(s), parte utilizada, indicação terapêutica (Tab. 1).

\section{Resultados e discussão}

A venda de plantas para uso medicinal e ritualístico no Município do Rio de Janeiro é uma prática difundida, relacionada em parte ao mercado formal, com feirantes e erveiros licenciados em suas respectivas barracas; e a um mercado informal onde erveiros (não licenciados pela Prefeitura do RJ) comercializam plantas em bancas isoladas e/ou diretamente nas calçadas.

Nas feiras livres das zonas Norte e Sul do RJ observou-se um número equivalente entre o mercado formal e o informal. Nestes locais, produtos e subprodutos das plantas são vendidas a partir dos seus nomes populares que muitas vezes pode interferir no processo de qualidade e fiscalização sanitária, pois não há registros explícitos dos processos de coleta, identificação e armazenamento.

A partir da técnica de Listagem Livre foram indicadas pelos erveiros 106 espécies úteis, distribuídas em 49 famílias botânicas e 92 gêneros. As seis famílias mais citadas foram Asteraceae (18 espécies), Lamiaceae (11 espécies), Leguminosae (seis espécies), Solanaceae, Poaceae e Verbenaceae (quatro espécies respectivamente) (Tab. 1). O hábito predominante foi o herbáceo $(56,1 \%)$, seguido do arbustivo $(28,5 \%)$, arbóreo $(11,4 \%)$ e trepadeira $(3,8 \%)$.

As entrevistas realizadas aos erveiros da zona Norte indicaram $57,5 \%$ do total das espécies úteis, sendo $45 \%$ relacionadas ao uso medicinal, $6,6 \%$ ritualísticas, e 5,6\% medicinal-ritual. Já os erveiros da zona Sul indicaram $42,5 \%$ de espécies, sendo $32 \%$ utilizadas como medicinal; 6,6\% ritualísticas; e 3,7\% como medicinal-ritual.

Nas feiras estudadas tanto da zona Norte quanto da zona Sul do RJ, o uso medicinal foi o mais representativo seguido do uso ritualístico. Ao longo deste estudo foram consideradas mais duas categorias, devido à relevância observada nas entrevistas, a categoria medicinal-alimentar representadas por (bredo - Tallinus patens (L.) Wild., pau-d'alho - Crataeva tapia L., jamelão - Syzygium cumini (L.) Skeels. e pitanga - Eugenia uniflora L.), ritual-alimentar (jambú - Acmella brachyolossa Cass. e manjericão - Ocimum basilicum L.) e apenas uma espécie exclusivamente alimentar (louro - Laurus nobilis L.).

No que se refere à parte utilizada encontrou-se um amplo uso das folhas na preparação dos remédios $(58 \%)$, seguido pelo uso da flor $(16,1 \%)$, a planta toda foi utilizada em $10,7 \%$ dos casos, e em porcentagem menores caule $(5,3 \%)$, frutos $(4,3 \%)$, casca $(3,2 \%)$ e raízes $(2,1 \%)$. Em relação à forma de utilização das plantas, $50 \%$ das espécies medicinais são consumidas como chás para beber, e 100\% das espécies ritualísticas na forma de banhos.

Comparando os resultados aqui apresentados com os realizados em outras feiras no Brasil, verificou-se que tanto em relação ao número de espécies quanto às espécies propriamente ditas, estes são semelhantes aos estudos realizados no Rio de Janeiro por Azevedo \& Silva (2006); M.M. Stalcup (dados não publicados); Parente \& Rosa (2001) (Tab. 2). Provavelmente, tal resultado relaciona-se com as espécies de ocorrência na Mata Atlântica, assim como o clima que propicia o cultivo de determinadas espécies, além dos aspectos culturais e tradicionais, especialmente pela influência européia que expressam o uso, extração e cultivo destas espécies comercializadas no Estado. E analisando os estudos realizados nas feiras e mercados na região Norte e Nordeste (Berg 1984; Pinto \& Maduro 2003; 
Tabela 1. Espécies úteis indicadas pelos erveiros das feiras livres das zonas Norte e Sul do Município do Rio de Janeiro, RJ, ordenadas por ordem alfabética de famílias botânicas (Uso: Al - alimentar, Med - medicinal, Ban - banho ritualístico; Parte da planta utilizada: Fo - folha, Fl - flor, Fr - fruto, Se - semente, Ca - casca, Rz - raiz, Pt - planta toda; Hab - hábito: Arb - arbóreo, Sub - subarbustivo, Her - herbáceo, Tre - trepadeira; N. Col. ${ }^{1}$ = Número referentes às coletas de V. Maioli-Azevedo; *espécie nativa).

\begin{tabular}{|c|c|c|c|c|c|}
\hline Família/Nome científico & Nome vulgar & Usos & Parte usada & $\mathrm{Hab}$ & N. Col. ${ }^{1}$ \\
\hline \multicolumn{6}{|l|}{ ALISMATACEAE } \\
\hline $\begin{array}{l}\text { Echinodorus grandiflorus (Cham. \& } \\
\text { Schltdl.) Micheli* }\end{array}$ & chapéu-de-couro & Ban, Med & Fo & Arb & VMA 145 \\
\hline \multicolumn{6}{|l|}{ ANACARDIACEAE } \\
\hline Mangifera indica $\mathrm{L}$. & mangueira & Med & Fo & Arb & VMA 157 \\
\hline Schinus terebinthifolius Raddi.* & aroeira & Ban & Fo & Arb & VMA 86 \\
\hline \multicolumn{6}{|l|}{ ANNONACEAE } \\
\hline Annona muricata L. & graviola & Med & Fo, Fr & Arb & VMA 88 \\
\hline Indeterminada 1 & guiné-caboclo & Ban & Fo & Her & VMA 146 \\
\hline \multicolumn{6}{|l|}{ APIACEAE } \\
\hline Foeniculum vulgare Mill. & erva-doce & Med & Fo & Her & VMA 35 \\
\hline \multicolumn{6}{|l|}{ APOCYNACEAE } \\
\hline Asclepia curassavica L. & oficial-de-sala & Ban & $\mathrm{Pt}$ & Her & VMA 56 \\
\hline \multicolumn{6}{|l|}{ ASTERACEAE } \\
\hline Achyrocline satureoides (Lam.) DC. * & macela & Med & Fo, Fl & Her & VMA 178 \\
\hline Acmella brachyolossa Cass.* & jambu & $\mathrm{Al}$, Ban & Fo, Fl & Her & VMA 43 \\
\hline Ageratum conyzoides $\mathrm{L}$. & erva -de-são-joão & Med & Fo & Sub & VMA 9 \\
\hline Arthemisia absinthium L. & losna & Med & Fo, Fr & Sub & VMA 158 \\
\hline Baccharis dracuntifolia DC. & alecrim-do-campo & Med & Fo, Fl & Sub & VMA 80 \\
\hline B. trimera (Less.) DC.* & carqueja & Med & $\mathrm{Ca}$ & Her & VMA 69 \\
\hline Bidens pilosa $\mathrm{L} . *$ & picão & Med & Fo & Her & VMA 176 \\
\hline Centratherum punctatum Cass.* & balainho de velho & Med & Fo, Fl & Her & VMA 147 \\
\hline Coreopsis grandiflore Hogg ex. Sweet. & camomila & Med & Fo & Her & VMA 177 \\
\hline Elephantopus angustifolius $\mathrm{Sw}$. & língua-de-vaca & Med & $\mathrm{Pt}$ & Her & VMA 183 \\
\hline Emilia coccinea Sims & dente-de-leão & Med & $\mathrm{Fo}, \mathrm{Fl}$ & Her & VMA 63 \\
\hline Helianthus annuus L. & girassol & Med & & Her & VMA 182 \\
\hline Mikania glomerata Spreng* & guaco & Med & Fo & Tre & VMA 55 \\
\hline Pterocaulon alopecuroides (Lam.)DC. & quitoco & Med & Fo & Her & VMA 160 \\
\hline Solidago chilensis Meyen & arnica & Med & Fo, Fl & Her & VMA 26 \\
\hline Tagetes erecta $\mathrm{L}$. & cravo & Ban & $\mathrm{Pt}$ & Her & VMA 41 \\
\hline Vernonia condensata Baker. & boldo & Med & Fo & Her & VMA 76 \\
\hline V. scabra Pers. & assa-peixe & Ban, Med & Fo & Sub & VMA 61 \\
\hline \multicolumn{6}{|l|}{ BIGNONIACEAE } \\
\hline Jacaranda sp.* & carobinha & Med & Fo & Arb & VMA 87 \\
\hline \multicolumn{6}{|l|}{ BIXACEAE] } \\
\hline Bixa orellana $\mathrm{L}$. & urucum & Med & $\mathrm{Se}, \mathrm{Fo}$ & Arb & VMA 157 \\
\hline \multicolumn{6}{|l|}{ BORAGINACEAE } \\
\hline $\begin{array}{l}\text { Cordia monosperma (Jacq.) Roem \& } \\
\text { Schult.* }\end{array}$ & baleeira-preta & Med & Fo, Fl & Arb & VMA 74 \\
\hline \multicolumn{6}{|l|}{ CAPPARACEAE } \\
\hline Crataeva tapia $\mathrm{L}^{*}$ & pau d'alho & Al, Med & $\mathrm{Fo}, \mathrm{Fr}, \mathrm{Ca}$ & Arb & VMA 148 \\
\hline \multicolumn{6}{|l|}{ CAPRIFOLIACEAE } \\
\hline Sambucus nigra L. & sabugueiro & Ban, Med & Fo,Fr & Arb & VMA 65 \\
\hline \multicolumn{6}{|l|}{ CHENOPODIACEAE } \\
\hline Chenopodium ambrosioides $\mathrm{L}$ & mastruz ou santa maria & Med & Fo & Her & VMA 36 \\
\hline \multicolumn{6}{|l|}{ CHRYSOBALANACEAE } \\
\hline Chrysobalanus icaco L.* & Abajiru & Med & Fo & Her & VMA 85 \\
\hline \multicolumn{6}{|l|}{ COSTACEAE } \\
\hline Costus spiralis (Jacq) Roscoe * & cana-do-brejo & Med & Fo & Her & VMA 161 \\
\hline \multicolumn{6}{|l|}{ CRASSULACEAE } \\
\hline Kalanchoe brasiliensis Cambess. & saião & Med & Fo & Her & VMA 49 \\
\hline K. pinnata (Lam.) Pers & fortuna & Ban & Fo & Her & VMA 48 \\
\hline \multicolumn{6}{|l|}{ CUCURBITACEAE } \\
\hline Momordica charantia L. & melão-de-são-caetano & Med & Fo, Fr & Her & $\begin{array}{l}\text { VMA } 32 \\
\text { continu }\end{array}$ \\
\hline
\end{tabular}


Tabela 1 (continuação)

\begin{tabular}{|c|c|c|c|c|c|}
\hline Família/Nome científico & Nome vulgar & Usos & Parte usada & $\mathrm{Hab}$ & N. Col. ${ }^{1}$ \\
\hline \multicolumn{6}{|l|}{ EQUISETACEAE } \\
\hline Equisetum hyemale L. & cavalinha & Med & $\mathrm{Pt}$ & Her & VMA 83 \\
\hline \multicolumn{6}{|l|}{ EUPHORBIACEAE } \\
\hline Acalypha poiretii Spreng. & parietária & Med & Fo & Arb & VMA 38 \\
\hline Jatropha gossypiifolia $\mathrm{L}$ & pinhão & Ban, Med & Fo & Her & VMA 59 \\
\hline Phyllanthus sp. & quebra-pedra & Med & Fo, Fl & Her & VMA 163 \\
\hline \multicolumn{6}{|l|}{ FLACOURTIACEAE } \\
\hline Casearia sylvestris $\mathrm{Sw} . *$ & são-gonçalino & Ban & Fo & Her & VMA 81 \\
\hline \multicolumn{6}{|l|}{ LAMIACEAE } \\
\hline Aeollanthus suaveolens Mart. ex Spreng. & macaça & Ban, Med & Fo, Fl & Her & VMA 58 \\
\hline Leonotis nepetifolia (L.) R.Br. & cordão-do-frade & Ban & $\mathrm{Pt}$ & Her & VMA 20 \\
\hline Leonurus sibiricus L. & macaé & Med & Fo, Fl & Her & VMA 16 \\
\hline Mentha gentilis L. & alevante & Ban, Med & Fo & Her & VMA 149 \\
\hline $\begin{array}{l}\text { Mentha subg. Pulegium (Mill.) Lam. \& } \\
\text { DC. ex Briq. }\end{array}$ & poejo & Med & Fo & Her & VMA 37 \\
\hline M. piperira var. citrata (Ehrh.) Briq. & elevante & Ban & $\mathrm{Pt}$ & Her & VMA 162 \\
\hline Ocimum basilicum $\mathrm{L}$. & manjericão & Ban, Med & Fo & Her & VMA 179 \\
\hline O. gratissimum $\mathrm{L}$. & alfavaca & Ban, Med & Fo, Fl & Sub & VMA 18 \\
\hline Plectranthus barbatus Andrews & malva & Med & Fo & Her & VMA 173 \\
\hline Rosmarinus officinalis L. & alecrim-de- horta & Ban, Med & Fo, Fl & Sub & VMA 73 \\
\hline Salvia officinalis L. & sálvia & Al, Ban & Fo & Sub & VMA 175 \\
\hline \multicolumn{6}{|l|}{ LAURACEAE } \\
\hline Cinnamomum zeylanicum Breyn. & canela & Ban, Med & $\mathrm{Fo}, \mathrm{Ca}$ & Arb & VMA 180 \\
\hline Laurus nobilis L. & louro & $\mathrm{Al}$ & Fo & Arb & VMA 174 \\
\hline \multicolumn{6}{|l|}{ LEGUMINOSAE } \\
\hline Bauhinia forficata Link. subsp. forficata * & pata-de-vaca 1 & Med & Fo & Her & VMA 14 \\
\hline B. radiata Vell $*$ & pata-de-vaca 2 & Med & Fo & Tre & VMA 181 \\
\hline B. microstachya Raddi* & pata-de-vaca 3 & Med & Fo & Tre & VMA 13 \\
\hline B. variegata $\mathrm{L}$. & pata-de-vaca 4 & Med & Fo & Arb & VMA 64 \\
\hline Desmodium adescendens (SW) DC. * & amor-do-campo & Med & Fo & Her & VMA 25 \\
\hline Mimosa pudica L. * & dormideira & Med & Fo & Tre & VMA 40 \\
\hline \multicolumn{6}{|l|}{ LILIACEAE } \\
\hline Aloe arborescens Mill. & babosa & Med & Fo & Her & VMA 172 \\
\hline \multicolumn{6}{|l|}{ LYTRACEAE } \\
\hline Cuphea carthagenesis (Jacq.) J.F.Macbr. & sete-sangrias & Med & Fo & Her & VMA 10 \\
\hline LORANTHACEAE & & & & & \\
\hline Struthanthus concinnus Mart. & erva-de- passarinho & Med & Fo & Tre & VMA 165 \\
\hline \multicolumn{6}{|l|}{ MALVACEAE } \\
\hline Gossypium barbadense L. & algodão & Ban, Med & Fo & Arb & VMA 57 \\
\hline Sida panicautis Cav. & vassorinha & Med & Fo & Sub & VMA 29 \\
\hline \multicolumn{6}{|l|}{ MELASTOMATACEAE } \\
\hline Miconia albicans (Sw.)Triana. * & abranda-fogo & Ban & Fo & Sub & VMA 78 \\
\hline \multicolumn{6}{|l|}{ MELIACEAE } \\
\hline Melia azedarach $\mathrm{L}$. & pára-raio & Ban & $\mathrm{Pt}$ & Arb & VMA 44 \\
\hline \multicolumn{6}{|l|}{ MONIMIACEAE } \\
\hline Siparuna guianensis Aubl. * & negra-mina & Ban & Fo, Fl, Fr & Arb & VMA 150 \\
\hline \multicolumn{6}{|l|}{ MORACEAE } \\
\hline Cecropia hololeuca Miq.* & embaúba - branca & Med & Fo & Arb & VMA 151 \\
\hline Morus nigra $\mathrm{L}$. & amora & Ban, Med & Fo, Fr & Arb & VMA 23 \\
\hline Sorocea guilleminiana Gaudich. * & espinheira-santa & Med & Fo & Arb & VMA 72 \\
\hline \multicolumn{6}{|l|}{ MUSACEAE } \\
\hline Heliconia psittacorum L.f. & lírio & Ban & Fo, Fl & Arb & VMA 70 \\
\hline \multicolumn{6}{|l|}{ MYRTACEAE } \\
\hline Eugenia uniflora L.* & pitanga & Al, Med & Fo, Fr & Arb & VMA 46 \\
\hline Syzygium cumini (L.) Skeels & jamelão & Al, Med & Fo & Arb & VMA 89 \\
\hline \multicolumn{6}{|l|}{ PHYTOLACACEAE } \\
\hline Petiveria alliacea $\mathrm{L}$. & guiné-piu-piu & Ban, Med & Fo & Sub & VMA 25 \\
\hline
\end{tabular}


Tabela 1 (continuação)

\begin{tabular}{|c|c|c|c|c|c|}
\hline Família/Nome científico & Nome vulgar & Usos & Parte usada & $\mathrm{Hab}$ & N. Col. ${ }^{1}$ \\
\hline \multicolumn{6}{|l|}{ PIPERACEAE } \\
\hline Peperomia pellucida (L.) Kunth.* & oriri & Ban, Med & Fo & Her & VMA 34 \\
\hline $\begin{array}{l}\text { Piper arborum var. falcifolium (Trel.) } \\
\text { Yunck.* }\end{array}$ & $\begin{array}{l}\text { aperta-ruão ou } \\
\text { vence-demanda }\end{array}$ & Ban, Med & Fo & Her & VMA 84 \\
\hline $\begin{array}{l}\text { P. hoffmann seggianum Roemer \& } \\
\text { Schultes.* }\end{array}$ & jaborandi & Med & Fo & Arb & VMA 45 \\
\hline \multicolumn{6}{|l|}{ PLANTAGINACEAE } \\
\hline Plantago major L. & transagem & Med & Fo & Her & VMA 152 \\
\hline \multicolumn{6}{|l|}{ POACEAE } \\
\hline Cenchrus ciliaris L. & capim-angola & Ban & Fo, Fl & Her & VMA 79 \\
\hline Coix lacrima-jobi $\mathrm{L}$ & lágrima-de-nossa-senhora & Ban, Med & Fo & Her & VMA 67 \\
\hline Cymbopogon citratus (DC) Stapf. & capim-limão & Med & Fo & Her & VMA 156 \\
\hline Zea mays L. & cabelo-de-milho & Med & Fo, Fl & Her & VMA 153 \\
\hline \multicolumn{6}{|l|}{ POLYGONACEAE } \\
\hline Antigonon leptopus Hook \& Arn. & amor-agarradinho & Ban & $\mathrm{Pt}$ & Tre & VMA 75 \\
\hline Polygon spectabilis L. & erva-de-bicho & Med & Fo & Her & VMA 176 \\
\hline \multicolumn{6}{|l|}{ PORTULACACEAE } \\
\hline Tallinum patens $(\mathrm{L})$. Willd & bredo & Al, Med & Fo & Her & VMA 62 \\
\hline \multicolumn{6}{|l|}{ PUNICACEAE } \\
\hline Punica granatum L. & romã & Med & Fo,Fr & Arb & VMA 166 \\
\hline \multicolumn{6}{|l|}{ RUBIACEAE } \\
\hline Coffea arabica $\mathrm{L}$. & café & Med & Fo, Fr & Sub & VMA 170 \\
\hline Spermacoce verticillata $\mathrm{L}$. & peito-de-moça & Med & Fo, Fl & Her & VMA 31 \\
\hline $\begin{array}{l}\text { Uncaria tomentosa (Willd. ex Roem. \& } \\
\text { Schult) DC. * }\end{array}$ & unha-de-gato & Med & Fo, Fl, Ra & Her & VMA 171 \\
\hline \multicolumn{6}{|l|}{ RUTACEAE } \\
\hline Ruta graveolens $\mathrm{L}$. & arruda & Ban, Med & $\mathrm{Pt}, \mathrm{Fo}$ & Her & VMA 167 \\
\hline \multicolumn{6}{|l|}{ SCHIZEAECEAE } \\
\hline Lygodium volubile Sw. & abre-caminho & Ban & Fo & Arb & VMA 53 \\
\hline \multicolumn{6}{|l|}{ SCROPHULARIACEAE } \\
\hline Scoparia dulcis L & $\begin{array}{l}\text { vassorinha-de- } \\
\text { nossa-senhora }\end{array}$ & Med & Fo & Her & VMA 30 \\
\hline \multicolumn{6}{|l|}{ SOLANACEAE } \\
\hline Cestrum laevigatum Schltdl. * & quairama & Med & Fo & Sub & VMA 39 \\
\hline Solanum americanum Mill. * & erva-moura & Ban, Med & $\mathrm{Pt}$ & Her & VMA 168 \\
\hline S. argenteum Dunal. * & erva-prata & Ban & Fo & Arb & VMA 82 \\
\hline S. paniculatum L.* & jurubeba & Med & Fo, Fl & Arb & VMA 52 \\
\hline \multicolumn{6}{|l|}{ STYRACACEAE } \\
\hline Styrax benzoin Dryand. & bejoim & Ban & Fo, Fl & Arb & VMA 54 \\
\hline \multicolumn{6}{|l|}{ URTICACEAE } \\
\hline Urera mitis L. & urtiga-branca & Ban, Med & Fo, Fl & Her & VMA 71 \\
\hline \multicolumn{6}{|l|}{ VERBENACEAE } \\
\hline Lantana camara $\mathrm{L}$. & cambará & Med & Fo, Fl & Arb & VMA 11 \\
\hline Lippia alba (Mill.) N.E.Br. * & erva-cidreira & Med & Fo & Her & VMA 33 \\
\hline $\begin{array}{l}\text { Stachytarpheta cayannensis (Rich.) } \\
\text { M.Vahl. * }\end{array}$ & gervão- roxo & Med & Fo & Her & VMA 7 \\
\hline Indeterminada 2 & alfazema & Ban & Her & Fo & VMA 42 \\
\hline \multicolumn{6}{|l|}{ VITACEAE } \\
\hline $\begin{array}{l}\text { Cissus verticillata (L.) Nicholson \& } \\
\text { C.E.Jarvis.* }\end{array}$ & insulina & Med & Fo & Ter & VMA 155 \\
\hline \multicolumn{6}{|l|}{ ZINGIBERACEAE } \\
\hline $\begin{array}{l}\text { Alpinia zerumbet (Pers.) B.L. Burtt \& } \\
\text { R.M. Sm. }\end{array}$ & colônia & Ban & Fo & Her & VMA 17 \\
\hline Zingiber officinale Roscoe. & gengibre & Med & $\mathrm{Ra}$ & Her & VMA 169 \\
\hline
\end{tabular}


Almeida \& Albuquerque 2002) verificou-se o fato contrário, ou seja, poucas espécies foram comuns, provavelmente por serem regiões de diferentes formações vegetais, bem como alta diversidade cultural, especialmente pela forte influência ainda indígena e africana existente (Tab. 2).

Do total das espécies identificadas, apenas 33\% são nativas e $67 \%$ são exóticas, provenientes da Europa e África. Tal resultado demonstra a forte influência da cultura européia, especialmente para o uso de plantas medicinais, e da africana para as plantas ritualísticas na região metropolitana do Rio de Janeiro.

Begossi et al. (2002) também registraram a forte influência européia em relação ao uso de plantas medicinais por caiçaras da Mata Atlântica, uma vez que das 227 espécies estudadas, apenas 38\% foram identificadas como nativas e as demais introduzidas e/ ou exóticas. Almeida (2003) salientou que nas regiões Sul e Sudeste do Brasil as plantas medicinais de origem européia são mais representativas, por terem se adaptado e difundido facilmente devido à presença de imigrantes.

A partir das entrevistas, observou-se um expressivo interesse não só das plantas medicinais, como as associadas a rituais. Este fato, já havia sido ressaltado nos levantamentos etnomédicos realizados por Almeida (2003) nas regiões metropolitanas do Rio de Janeiro e Salvador, onde registrou intenso consumo de espécies vegetais (de origem africana) através de terreiros afro-brasileiros, sendo estas plantas compradas em barracas de mercados populares e/ou erveiros de rua.

Ao compararmos as partes das plantas utilizadas neste estudo com as indicadas por Parente \& Rosa (2001), Almeida \& Albuquerque (2002) e Pinto \&
Maduro (2003), embora estes estudos tenham demonstrado proximidade em termos de diversidade de espécies, em relação às partes utilizadas dos vegetais, não foi encontrada semelhança. Esse resultado pode ser relacionado, ao fato das espécies serem diferentes em cada região, mudando assim, o modo de utilização dessas plantas pelos erveiros.

Relacionou-se 29 indicações terapêuticas, sendo o banho ritualístico a categoria de uso mais citada (138 citações), seguida por problemas no pulmão (70 citações) e problemas no estômago (30 citações). As indicações terapêuticas indicadas pelos erveiros das zonas Norte e Sul também foram semelhantes às de Parente \& Rosa (2001), pois a indicação para banhos ritualísticos foi alta $(66,6 \%)$, seguido de problemas respiratórios e problemas na pele. Já Almeida \& Albuquerque (2002), em Pernambuco, obtiveram resultados distintos, com maior número de usos reportados as espécies usadas em problemas respiratórios, seguidos por transtornos do sistema circulatório e sistema nervoso. Pinto \& Maduro (2003), encontraram maior uso de plantas para inflamações diversas, seguido de gripe e tratamento de malária. Esse resultado pode estar relacionado à regionalidade das doenças, ou seja, as doenças, assim como as espécies, variam de região para região afetando e caracterizando o comércio local de plantas medicinais.

O banho ritualístico, de descarrego, para induzir o bem-estar, além de afastar maus espíritos e mau olhado, foi uma prática relevante neste estudo por apresentar um número significativo de espécies (exclusivas para este fim) e alto número de citações (138). Verger (1995), entre outros autores desenvolveram trabalhos relacionados a estas formas de utilização destacando a importância de uma investigação de caráter

Tabela 2. Comparação das informações compiladas de estudos realizados em feiras livres no Brasil, em ordem cronológica de publicação. (№ Info - Número de informantes em cada trabalho; P.U. - Parte da planta mais utilizada em cada trabalho; C. Uso - Categoria de uso mais citada em cada trabalho; I. Ter. -Indicação terapêutica mais citada em cada trabalho; №. Esp. - Número de espécies totais encontrada em cada trabalho; № Esp. comuns - Número de espécies em comum com o presente estudo; Fo - folha; Ca - casca; Pt - planta toda; Me - espécies medicinais; Ri - espécies ritualística; Ba.- banhos ritualísticos; Re- problemas respiratórios; In - inflamações gerais).

\begin{tabular}{|c|c|c|c|c|c|c|c|}
\hline Fonte & Local & № $\operatorname{Inf}$ & P.U. & C. Uso & I. Ter. & № Esp. & № Esp. comuns \\
\hline Berg 1984 & Belém, PA & - & - & $\mathrm{Me}$ & - & 163 & 10 \\
\hline Stalcup 2000 & Rio de Janeiro, RJ & 5 & Fo & $\mathrm{Me} / \mathrm{Ri}$ & $\mathrm{Ba}$ & 151 & 62 \\
\hline Parente \& Rosa 2001 & Barra do Pirái, RJ & 2 & $\mathrm{Pt}$ & $\mathrm{Me}$ & $\mathrm{Ba}$ & 100 & 33 \\
\hline Almeida \& Albuquerque 2002 & Caruaru, PE & 20 & $\mathrm{Ca} / \mathrm{Fo}$ & $\mathrm{Me}$ & $\operatorname{Re}$ & 114 & 24 \\
\hline Pinto \& Maduro 2003 & Boa Vista, RO & 5 & $\mathrm{Ca} / \mathrm{Fo}$ & $\mathrm{Me}$ & In & 117 & 16 \\
\hline Azevedo \& Silva 2006 & Rio de Janeiro, RJ & 29 & - & $\mathrm{Me} / \mathrm{Ri}$ & - & 133 & 64 \\
\hline Presente estudo & Rio de Janeiro, RJ & 54 & Fo & $\mathrm{Me} / \mathrm{Ri}$ & $\mathrm{Ba}$ & 106 & - \\
\hline
\end{tabular}


farmacobotânico em função dos princípios ativos, responsáveis pelos efeitos que causam àqueles que delas se utilizam.

A partir das entrevistas realizadas, pode-se verificar o modo de obtenção das 106 plantas indicadas, sendo que $66 \%$ dos erveiros compram suas plantas em grandes mercados da cidade, como CEASA, Mercadão de Madureira e CADEG; $14 \%$ cultivam e $20 \%$ extraem da mata de diversos pontos da cidade.

A forma de obtenção das plantas indicadas pelos erveiros tanto da zona Norte quanto da zona Sul, foi bastante similar, pois na maioria destes erveiros as plantas são compradas em grandes mercados da cidade (CEASA, Mercadão de Madureira e o CADEG) e depois repassadas nas feiras livres da cidade do RJ. Tal prática dificultou a análise do volume de plantas extraídas de áreas naturais, pois apenas $20 \%$ dos erveiros totais entrevistados, informaram que extraem plantas da Floresta da Tijuca, Mata do Grajaú, Tinguá, Magé, entre outros. Estas informações também foram relatadas por Parente \& Rosa (2001), sendo que neste caso as extrações foram das matas de Barra do Piraí (25\% das plantas vendidas foram provenientes de extrativismo). Já Azevedo \& Silva (2006), encontraram valores relevantes de extração de plantas nas feiras e mercados da zona Oeste do Rio de Janeiro, pois relataram que $48,8 \%$ das espécies medicinais e ritualísticas vendidas foram originárias de extrativismo predatório.

Em relação à aplicação da fórmula de importância relativa, foram ressaltadas seis espécies (Bidens pilosa L., Cinnamomum zeylanicum Breyn., Echinodorus grandiflorus (Cham. \& Schltdl.) Micheli, Piper hoffmann seggianum Roemer \& Schultes., Ruta graveolens L., Uncaria tomentosa (Willd. ex Roem. \& Schult) DC.) sendo estas as mais versáteis, com múltiplos usos que atingiram até cinco categorias de uso relacionadas (Tab. 4).

De acordo com o consenso dos informantes das feiras livres das zonas Norte e Sul do RJ, quanto á potencialidade das espécies de plantas citadas, as indicações terapêuticas que atingiram o valor máximo de consenso $(\mathrm{FCI}=1)$ foram às categorias relacionadas a Neoplasias (câncer) e Transtornos Mentais e Comportamentais (Depressão). Esses valores indicam que essas categorias são culturalmente importantes e merecem estudos mais aprofundados (Tab. 3). Salienta-se a possível limitação do índice de Trotter \& Logan (1986), pois este pode vir a supervalorizar determinadas categorias, como as encontradas neste estudo, devido ao baixo número de citações e espécies envolvidas que permitiu atingir o valor máximo de consenso para nas duas categorias citadas. Tal fato, sobre a possível supervalorização de dados a partir da aplicação deste índice, pode ser observado também no estudo de Almeida \& Albuquerque (2002), cuja categoria de uso em destaque foi a de transtornos do sistema sensorial (ouvido), obtida a partir do valor máximo de FCI (sistema sensorial) que apresentou o valor mais baixo de número de espécie (uma espécie), com apenas dois usos reportados a essa referida espécie. Com isto, refletindo sobre os respectivos resultados obtidos a partir do uso do índice de Trotter \& Logan (1986), sugere-se maior atenção na análise dos resultados, pois tal índice tende a valorizar espécies pouco indicadas.

Ao comparar as espécies indicadas pelos erveiros das zonas Norte e Sul, com a Lista de Espécies Ameaçadas do IBAMA (1992), observou-se que não há espécies em perigo ou ameaçadas de extinção. Tal fato, provavelmente está relacionado ao hábito, uma vez que as espécies registradas no documento do IBAMA apresentam porte arbóreo, e as indicadas no presente estudo na maioria são herbáceas.

O extrativismo predatório é uma prática significativa que leva a perpetuação de um mercado clandestino de plantas medicinais e/ou ritualísticas. Isto dificulta a obtenção de dados fidedignos sobre a procedência das plantas medicinais e/ou ritualísticas vendidas nas feiras livres e mercados. Em seu estudo nas feiras livres e mercados no RJ, Azevedo \& Silva (2006), obtiveram 48,8\% do material botânico oriundo de extrativismo, sendo que $54,9 \%$ das plantas ocorrem em Ambientes de Mata Atlântica. Conseqüentemente, destacam-se a importância do estabelecimento de linhas de ação voltadas para o desenvolvimento, conservação e manejo, visando à utilização sustentável destas espécies.

Ressalta-se que o sistema terapêutico dos erveiros nas feiras livres do RJ é dinâmico e aberto a influências externas (eg. a mídia através de telejornais, revistas), alterando o caráter tradicional desse conhecimento. As indicações terapêuticas, assim como as partes da plantas mais utilizadas em feiras livres, provavelmente podem alterar conforme a região estudada, porém torna-se necessário e urgente uma maior coleta de dados padronizados nas feiras livres e mercados para melhor inferir sobre este resultado.

Os erveiros em determinados casos indicam e vendem plantas medicinais que ainda não tem validação, e/ou não tiveram seus compostos químicos testados, análises toxicológicas finais, como por exemplo, a venda 
Tabela 3. Indicações terapêuticas encontradas nas feiras livres das zonas Norte e Sul do município do Rio de Janeiro, RJ, adaptadas da Classificação Estatística Internacional para Doenças e Problemas relacionados à Saúde da OMS (1996), ordenada por número de citações (№ de citações - número de vezes que determinada categoria foi citada, № de espécies - número de espécies indicadas para determinada categoria, $\mathrm{FCI}=$ fator de consenso dos informantes).

\begin{tabular}{|c|c|c|c|}
\hline Categoria de indicações terapêuticas & № de citações & № de espécies & FCI \\
\hline Neoplasia & 2 & 1 & 1 \\
\hline Transtornos mentais e comportamentais (depressão) & 5 & 1 & 1 \\
\hline Doenças dos olhos (conjuntivite, coceira) & 11 & 2 & 0,9 \\
\hline Problemas no cabelo & 24 & 4 & 0,86 \\
\hline Doenças do sistema endócrino (diabetes) & 21 & 4 & 0,85 \\
\hline Anti-inflamatório (geral) & 21 & 4 & 0,85 \\
\hline Doenças do aparelho respiratório (asma, bronquite, tuberculose, gripe) & 70 & 12 & 0,84 \\
\hline Banhos ritualísticos (descarrego, astral, limpeza) & 138 & 26 & 0,81 \\
\hline Anti-pirético (febre) & 2 & 5 & 0,75 \\
\hline Doenças nutricionais e metabólicas (colesterol) & 5 & 2 & 0,75 \\
\hline Anti-hipertensivo (pressão slta) & 9 & 3 & 0,75 \\
\hline Analgésico (dores não definidas) & 7 & 3 & 0,66 \\
\hline Doenças do sistema osteomuscular (artrose e reumatismo) & 4 & 6 & 0,6 \\
\hline Anti-helmíntico & 10 & 5 & 0,55 \\
\hline Abortiva & 3 & 2 & 0,5 \\
\hline Anti-diarrêico & 3 & 2 & 0,5 \\
\hline Efeito sedativo (calmante) & 9 & 5 & 0,5 \\
\hline Doenças do aparelho circulatório (coração) & 3 & 2 & 0,5 \\
\hline Banhos medicinais (coceiras, manchas na pele, lavagens para afecções femininas) & 16 & 9 & 0,46 \\
\hline Doenças do aparelho digestivo (hepatite e indigestão) & 35 & 21 & 0,4 \\
\hline Doenças do aparelho geniturinário (diurético, pedra nos rins) & 18 & 12 & 0,35 \\
\hline Cicatrizantes & 4 & 3 & 0,33 \\
\hline Emagrecer & 4 & 3 & 0,33 \\
\hline Doenças do sangue e órgãos hematopoéticos (anemia e circulação) & 4 & 5 & 0,25 \\
\hline Emenagoga & 1 & 1 & 0 \\
\hline Menopausa & 2 & 2 & 0 \\
\hline Tônico & 2 & 2 & 0 \\
\hline
\end{tabular}

de Sorocea guilleminiana e Bauhinia variegata. Neste caso, seria interessante criar estratégias de aproximação entre erveiros e instituições de pesquisa para proporcionar maior troca de informações tanto científicas como empíricas, para que os produtos vendidos tenham melhor qualidade para o consumidor, bem como orientações relacionadas à forma de obtenção destas plantas, pois muitas vêm sofrendo com o forte processo de extrativismo.

Almeida (2003) ressalta que algumas espécies vegetais produzem substâncias tóxicas e pode-se relacionar que cerca de $25,5 \%$ das plantas indicadas pelos erveiros nas feiras estão registradas na Lista de Plantas Tóxicas do PROPLAM e do Center for Food Safety \& Applied Nutrition (CFAN), e 15\% destas espécies possuem contra-indicações para gestantes e mulheres que estão amamentando (SUS 2004). Ou seja, é urgente a criação de estratégias que orientem, especialmente os erveiros que tem contato direto com os consumidores, para garantir melhor qualidade dos produtos vendidos nas feiras livres.

No Brasil muitos estudos sobre plantas medicinais, seus usos e princípios ativos vêm sendo desenvolvidos há muitos anos e em diferentes áreas de conhecimento (botânica, farmacologia, agronomia, entre outras). Entretanto há pouca difusão e divulgação destes resultados obtidos para a população em geral e consumidora destas plantas. Torna-se urgente o incremento de estudos interdisciplinares para difusão destes conhecimentos, principalmente no sentido de informar e alertar sobre os possíveis males causados por determinadas plantas ou grupos vegetais à população.

Ressalta-se a necessidade do desenvolvimento de estudos sobre comércio de plantas medicinais nativas, assim como sobre cultivo e validação destas plantas para utilização como medicamentos, ou aproveitá-as adequada e economicamente de forma regional e nacional (TRAFFIC 2001).

Informações obtidas de estudos desta natureza podem vir a ser aplicados em ações direcionadas a 
Tabela 4. Valores de importância relativa (IR) de cada espécie vegetal utilizada como medicinal e ritualística pelos erveiros das feiras livres das zonas Norte e Sul do município do RJ, organizada por ordem decrescente de IR.

\begin{tabular}{|c|c|c|c|}
\hline Nome científico & IR & Nome científico & IR \\
\hline Bidens pilosa $\mathrm{L}$. & 1,2 & Centratherum punctatum Cass. & 0,4 \\
\hline Cinnamomum zeylanicum Breyn. & 1,2 & Cestrum laevigatum Schltdl. & 0,4 \\
\hline Echinodorus grandiflorus (Cham. \& Schltdl.) Micheli & 1,2 & Chrysobalanus icaco L. & 0,4 \\
\hline Piper hoffmann seggianum Roemer \& Schultes. & 1,2 & Cissus verticillata (L.) Nicholson \& C.E. Jarvis. & 0,4 \\
\hline Ruta graveolens $\mathrm{L}$. & 1,2 & Coix lacrima-jobi $\mathrm{L}$. & 0,4 \\
\hline Uncaria tomentosa (Willd. ex Roem. \& Schult) DC. & 1,2 & Cordia monosperma (Jacq.) Roem \& Schult. & 0,4 \\
\hline Aeollanthus suaveolens Mart. ex Spreng. & 1 & Coreopsis grandiflore Hogg ex. Sweet. & 0,4 \\
\hline Crataeva tapia $\mathrm{L}$. & 1 & Cuphea carthagenesis (Jacq.) J.F. Macbr. & 0,4 \\
\hline Ocimum gratissimum $\mathrm{L}$. & 1 & Cymbopogon citratus (DC.) Stapf. & 0,4 \\
\hline Petiveria alliacea $\mathrm{L}$. & 1 & Desmodium adescendens (Sw.) DC. & 0,4 \\
\hline Schinus terebinthifolius Raddi. & 1 & Elephantopus angustifolius Sw. & 0,4 \\
\hline Sida panicautis Cav. & 1 & Equisetum hyemale $\mathrm{L}$. & 0,4 \\
\hline Sorocea guilleminiana Gaudich. & 1 & Eugenia uniflora $\mathrm{L}$. & 0,4 \\
\hline Aloe arborescens Mill. & 0,8 & Foeniculum vulgare Mill. & 0,4 \\
\hline Alpinia zerumbet (Pers.) B.L. Burtt \& R.M. Sm. & 0,8 & Gossypium barbadense L. & 0,4 \\
\hline Arthemisia absinthium L. & 0,8 & Helianthus annuus L. & 0,4 \\
\hline Baccharis dracuntifolia DC. & 0,8 & Heliconia psittacorum L. f. & 0,4 \\
\hline Bixa orellana $\mathrm{L}$. & 0,8 & Jatropha gossypiifolia $\mathrm{L}$. & 0,4 \\
\hline Cenchrus ciliaris L. & 0,8 & Kalanchoe brasiliensis Cambess. & 0,4 \\
\hline Chenopodium ambrosioides $\mathrm{L}$ & 0,8 & Kalanchoe pinnata (Lam.) Pers & 0,4 \\
\hline Emilia coccinea Sims & 0,8 & Lantana camara $\mathrm{L}$. & 0,4 \\
\hline Leonurus sibiricus $\mathrm{L}$. & 0,8 & Laurus nobilis L. & 0,4 \\
\hline Melia azedarach $\mathrm{L}$. & 0,8 & Leonotis nepetifolia (L.) R. Br. & 0,4 \\
\hline Miconia albicans (Sw.) Triana. & 0,8 & Lippia alba (Mill.) N.E. Br. & 0,4 \\
\hline Ocimum basilicum $\mathrm{L}$. & 0,8 & Lygodium volubile $\mathrm{Sw}$. & 0,4 \\
\hline Rosmarinus officinalis $\mathrm{L}$. & 0,8 & Mangifera indica $\mathrm{L}$. & 0,4 \\
\hline Sambucus nigra L. & 0,8 & Mentha gentilis $\mathrm{L}$. & 0,4 \\
\hline Stachytarpheta cayannensis (Rich.) M. Vahl. & 0,8 & Mentha piperira var. citrata (Ehrh.) Briq. & 0,4 \\
\hline Tallinum patens (L.). Willd & 0,8 & Mentha subg. Pulegium (Mill.) Lam. \& DC. ex Briq. & 0,4 \\
\hline Vernonia condensata Baker. & 0,8 & Mikania glomerata Spreng & 0,4 \\
\hline Zingiber officinale Roscoe. & 0,8 & Mimosa pudica L. & 0,4 \\
\hline Ageratum conyzoides $\mathrm{L}$. & 0,6 & Morus nigra $\mathrm{L}$. & 0,4 \\
\hline Baccharis trimera (Less.) DC. & 0,6 & Peperomia pellucida (L.) Kunth. & 0,4 \\
\hline Coffea arabica $\mathrm{L}$. & 0,6 & Phyllanthus sp. & 0,4 \\
\hline Jacaranda sp. & 0,6 & Piper arborum var. falcifolium (Trel.) Yunck. & 0,4 \\
\hline Momordica charantia $\mathrm{L}$. & 0,6 & Plectranthus barbatus Andrews & 0,4 \\
\hline Plantago major $\mathrm{L}$. & 0,6 & Polygon spectabilis $\mathrm{L}$. & 0,4 \\
\hline Solanum americanum Mill. & 0,6 & Pterocaulon alopecuroides (Lam.) DC. & 0,4 \\
\hline Solanum paniculatum $\mathrm{L}$. & 0,6 & Punica granatum $\mathrm{L}$. & 0,4 \\
\hline Costus spiralis (Jacq) Roscoe & 0,41 & Salvia officinalis L. & 0,4 \\
\hline Acalypha poiretii Spreng. & 0,4 & Scoparia dulcis L. & 0,4 \\
\hline Achyrocline satureoides (Lam.) DC. & 0,4 & Siparuna guianensis Aubl. & 0,4 \\
\hline Acmella brachyolossa Cass. & 0,4 & Solanum argenteum Dunal. & 0,4 \\
\hline Annona muricata $\mathrm{L}$. & 0,4 & Solidago chilensis Meyen & 0,4 \\
\hline Antigonon leptopus Hook \& Arn. & 0,4 & Spermacoce verticillata $\mathrm{L}$. & 0,4 \\
\hline Asclepia curassavica $\mathrm{L}$. & 0,4 & Struthanthus concinnus Mart. & 0,4 \\
\hline Bauhinia forficata Link. subsp. forficata & 0,4 & Styrax benzoin Dryand. & 0,4 \\
\hline Bauhinia microstachya Raddi & 0,4 & Syzygium cumini (L.) Skeels & 0,4 \\
\hline Bauhinia radiata Vell & 0,4 & Tagetes erecta $\mathrm{L}$. & 0,4 \\
\hline Bauhinia variegata $\mathrm{L}$. & 0,4 & Urera mitis L. & 0,4 \\
\hline Casearia sylvestris $\mathrm{Sw}$. & 0,4 & Vernonia scabra Pers. & 0,4 \\
\hline Cecropia hololeuca Miq. & 0,4 & Zea mays L. & 0,4 \\
\hline
\end{tabular}


orientação tanto à erveiros quanto à consumidores de plantas, fornecer informações relevantes para a Vigilância Sanitária e órgãos de fiscalização; e especialmente subsidiar estudos de conservação e manejo de espécies medicinais.

\section{Agradecimentos}

Aos erveiros das zonas Norte e Sul do Município do Rio de Janeiro por compartilharem seus conhecimentos; aos botânicos: Ângela Vaz, Alexandre Quinet, Elsie F. Guimarães, R.M. Harley, Inês Machline, João Marcelo A. Braga, Marcela S. Kropf, Massimo G. Bovini, Ravena Dias de Melo, Regina H. P. Andreata, Roberto Esteves, Robson D. Ribeiro,Vidal Mansano, pela identificação taxonômica do material encontrado nas feiras livres; Thiago M. Azevedo pelo mapa, Isa Maioli pela revisão do texto e Dra. Christina Paixão pela ajuda com a tabela três.

\section{Referências bibliográficas}

Alexiades, M.N. 1996. Selected guidelines for ethnobotanical research: a field manual. New York, The New York Botanical Garden Press.

Almeida, M.Z. 2003. Plantas medicinais. 2 ed. Salvador, EDUFBA.

Almeida, C.F.C.B. \& Albuquerque, U.P. 2002. Uso e conservação de plantas e animais medicinais no estado de Pernambuco (Nordeste do Brasil): um estudo de caso. Interciência 26(6): 276-285.

Akerele, O. 1998. Medicinal plants and primary health care: an agenda for action. Fitoterapia 59: 355-363.

Azevedo, S.K.S. \& Silva, I. M.2006. Comercialização de plantas medicinais e de uso religioso no município do Rio de Janeiro. Acta Botanica Brasilica 20(1): 185-194.

Balick, M.J.; Kronenberg, F.; Ososki, A.L.; Reiff, M.; FughBerman, A.; O'Connor, B.; Roble, M.; Lohr, P. \& Atha, D. 2000. Medicinal plants used by Latino healers for women's health Conditions. New York City. Economic Botany 54(3): 344-357.

Begossi, A.; Hanazaki, N. \& Tamashiro,J.Y. 2002. Medicinal plants in the atlantic forest (Brazil): knowledge, use, and conservation. Human Ecology 30(3): 281-299.

Bennett, B.C. \& Prance, G.T. 2000. Introduced plants in indigenous pharmacopoeia of Northern South America. Economic Botany 54: 90-102.

Berg, M.E.V.D. 1984. Ver-o-peso: The Ethonobotany of an Amazonain Market. In: Advances in Ecomonic Botany. G.T. Prance \& Kallunki. v. 1. The New York Botanical Garden.

Bernard, R.H. 1989. Research methods in cultural anthropology. Londres, SAGE Publications, Inc.

Bodeker, G.C. 1994. Global health tradicions. Pp. 279-290. In: M.S. Micozzi, ed. (1996). Fundamentals of complementary and alternative medicine. New York.
Camarra, D. 1995. O pensamento médico popular. Ribro Soft, Ed. e Informática Ltda. RJ-Brasil.

Center for Food Safety \& Applied Nutrition (CFAN) Poisonous Plant Database http://vm.cfsan.fda.gov/ $\sim$ djw/plantnam.html (Acesso: 22/11/2004).

Cotton, C.M. 1996. Ethonbotany: principles and applications. England, Wiley.

Cunningham, A.B. 1993. Ethics, etnobiological research, and biodiversity. WWF International publications. B. Lindsay (ed.). Gland, Switzerland.

Gorberg, S. \& Fridman, S.A. 2003. Mercados no Rio de Janeiro - 1834-1962. Rio de Janeiro.

Gottlieb, O.R.; BORIN, M.R.M.B.; Pagotto, C.L.A.C. \& Zocher, D. H.T. 1998. Biodiversidade: o enfoque interdisciplinar brasileiro. Ciência \& Saúde Coletiva 3(2): 97-102.

IBAMA. 1992. Lista oficial de espécies da flora brasileira ameaçada de extinção. Anexo 2, Portaria n 37-N.

IUCN 1993. Guidelines on the conservation of medicinal plants. IUCN, WHO and WWF, Gland, Switzerland.

Martin, G.J. 1995. Ethobotany - A method manual. New York, Chapman \& Hall.

Organização Mundial de Saúde (OMS). 1996. Classificação estatística internacional de doenças e problemas relacionados à saúde. Décima revisão. Edusp.

Organização Mundial de Saúde (OMS). 2002. Estratégia de la OMS sobre medicina tradicional 2002-2005.

Parente, C.E.T \& Rosa, M.M.T. 2001. Plantas comercializadas como medicinal no Município de Barra do Piraí, RJ. Rodriguésia 52(80): 47-59.

Pinto, A.A.C. da \& Maduro, C.B. 2003. Produtos e subprodutos da medicina popular comercializados na cidade de Boa Vista, Roraima. Acta Amazônica 33(2): 281-290.

Prefeitura do Estado do Rio de Janeiro. 2003. http:// www.rio.rj.gov.br/clf/feiras/feirart.htm (Acesso: 22/04/2003).

PROPLAM - Programa de Plantas Medicinais. 2000. Fitoterapia na assistência à Gestante. Secretaria de Saúde do Estado. Rio de Janeiro, RJ.

Reis, M.S. 1996. Manejo sustentado de plantas medicinais em ecossistemas tropicais. Pp. 199-215. In: Plantas Medicinais: arte e ciência - um guia multidisciplinar. L.C. Di Stasi, (org.). São Paulo, Ed. Unesp.

Santos, M.G. \& Silvestre, L.S. 2000. Pteridófitas comercializadas por erveiros de Niterói e do Rio de Janeiro, RJ, Brasil: Uma abordagem etnobotânica. Leandra 15: 79-90.

SAREM/ SEPLAN - RR. 1982. O que é preciso saber sobre mercados e feiras livres. Rio de Janeiro.

Shanley, P.; Luz, L. \& Swingland. 2002. The faint promise of a distant market: a survey of Belem's trade in non timber forest products. Biodiversity and Conservation 11: 615-632.

Simões, C.M.O.; Mentz, L.E.; Schenkel, E.P.; Irgang, B.E. \& Stehmann, J.R. 1998. Plantas medicinais populares no Rio Grande do Sul. 3. ed. Porto Alegre, Ed. Univers.

Sistema Único de Saúde (SUS). 2004. Secretária de Saúde do Estado do Rio de Janeiro. http://www.saude.rj.gov.br/ (Acesso: 11/2004). 
TRAFFIC. 2001. Plantas medicinais do Brasil: aspectos gerais sobre a legislação e Comércio. WWF/IBAMA.

Trotter, R.T. \& Logan, M.H. 1986. Informant consensus: a new approach for identifying potentially effective medicinal plants. Pp. 91-112. In: N.L. Etkin (ed.). Plants in indigenous medicine and diet: biobehavioral approachs. New York, Redgrave Publishing C.
Weller, S.C. \& Kimball-Romney, A. 1988. Systematic data collection. Newbury, SAGE.

World Health Organization. 2002. General guidelines for methodologies on research and evaluation of tradicinal medicine. Geneva.

Verger, P.F. 1995. Ewé: o uso das plantas na sociedade ioruba. São Paulo, Companhia das Letras. 